\title{
Atypical clinical presentation of a subset of patients with anti-RNA polymerase III - non- scleroderma cases associated with dominant RNA polymerase I reactivity and nucleolar staining
}

Angela Ceribelli ${ }^{1}$, Malgorzata E Krzyszczak ${ }^{2}$, Yi Li $^{2}$, Steven J Ross ${ }^{1,2}$, Jason YF Chan ${ }^{2}$, Edward KL Chan ${ }^{1}$, Rufus W Burlingame ${ }^{3}$, Tyler T Webb ${ }^{3}$, Michael R Bubb², Eric S Sobel ${ }^{2,4}$, Westley H Reeves ${ }^{2,4}$ and Minoru Satoh ${ }^{2,4^{*}}$

\begin{abstract}
Introduction: Anti-RNA polymerase III (RNAP III) antibodies are highly specific markers of scleroderma (systemic sclerosis, SSc) and associated with a rapidly progressing subset of SSc. The clinical presentation of anti-RNAP III positive patients, onset of Raynaud's phenomenon (RP) and SSC in unselected patients in a rheumatology clinic were evaluated.

Methods: Autoantibodies in sera from 1,966 unselected patients (including 434 systemic lupus erythematosus (SLE), 119 SSc, 85 polymyositis/dermatomyositis (PM/DM)) in a rheumatology clinic were screened by radioimmunoprecipitation. Anti-RNAP III positive sera were also tested by immunofluorescence antinuclear antibodies and anti-RNAP III ELISA. Medical records of anti-RNAP III positive patients were reviewed.
\end{abstract}

Results: Among 21 anti-RNAP III positive patients, 16 met the American College of Rheumatology (ACR) SSc criteria at the initial visit but 5 did not; diagnoses were vasculitis, early polyarthritis, renal failure with RP, interstitial lung disease, and Sjögren's syndrome. The first two patients developed rapidly progressive diffuse SSc. An additional case presented with diffuse scleroderma without RP and RP developed two years later. Anti-RNAP III antibodies in these 6 cases of atypical clinical presentation were compared with those in 15 cases of typical (SSc with RP) cases. Anti-RNAP III levels by ELISA were lower in the former group ( $P=0.04$ by Mann-Whitney test) and 3 of 6 were negative versus only 1 of 15 negative in the latter $(P<0.05$ by Fisher's exact test). Three cases of non-SSc antiRNAP III positive patients had predominant reactivity with RNAP I with weak RNAP III reactivity and had a strong nucleolar staining. Three anti-RNAP III patients, who did not have RP at the initial visit, developed RP months later. Scleroderma developed prior to RP in 5 out of $16(31 \%)$ in the anti-RNAP III group, but this was rare in patients with other autoantibodies. The interval between the onset of RP to scleroderma was short in anti-RNAP III positive patients.

Conclusions: Anti-RNAP III antibodies are highly specific for SSc; however, a subset of anti-RNAP III positive patients do not present as typical SSc. The interval between RP and scleroderma in this group is short, and 31\% of patients developed scleroderma prior to RP in this group. Anti-RNAP III positive patients may not present as typical SSC and detecting anti-RNAP III may have predictive value.

\footnotetext{
* Correspondence: minoru.satoh@medicine.ufl.edu

2Division of Rheumatology and Clinical Immunology, Department of Medicine, University of Florida, 1600 SW Archer Road, Gainesville, FL 32610-

0221, USA

Full list of author information is available at the end of the article
} 


\section{Introduction}

Specific autoantibodies in systemic rheumatic diseases are useful biomarkers associated with certain diagnoses and/or clinical manifestations [1]. Several autoantibodies, including anti-topoisomerase I (topo I), -centromere (ACA), -RNA polymerase III (RNAP III), -U3RNP/ fibrillarin, and -Th/To, have been reported to be associated with scleroderma (systemic sclerosis, SSc); some are considered highly specific disease markers while others are considered relatively specific [2]. Anti-RNAP III that is considered highly specific for SSc, is a relatively new disease marker of SSc; however, it has become a popular test in the last several years thanks to the wide availability of commercial ELISA kits $[1,2]$. Detecting anti-RNAP III in some undiagnosed patients would not be totally unexpected, considering that autoantibodies are usually produced prior to typical clinical manifestations [3]. However, detection of anti-RNAP III in non-SSc patients or prior to clinical SSc has rarely been reported [4]. Although anti-RNAP III antibodies are associated with rapid progression of the disease and the interval between the onset of Raynaud's phenomenon (RP) and SSc is short [2,5], the time course of the onset of RP and SSc has not been well described. In the present study, the clinical features of anti-RNAP III positive patients in a cohort of an unselected population in a rheumatology clinic that includes undiagnosed patients and patients with a wide variety of diagnosis, were characterized. The relationships among detection of anti-RNAP III antibodies, onset of RP, and development of sclerodermatous skin changes, were also systematically analyzed.

\section{Materials and methods Patients}

All 1,966 subjects enrolled in the University of Florida Center for Autoimmune Diseases (UFCAD) registry from 2000 to 2010 were studied. Diagnoses of the patients include 434 SLE, 119 SSc, 85 polymyositis/dermatomyositis, and various other diagnoses, and many remained undiagnosed for a specific systemic autoimmune disease. At each visit of the enrolled subjects, a form with a standard check list of symptoms and physical findings, including Raynaud's phenomenon and sclerodermatous skin changes, was filled out by physicians in addition to an entry in the medical chart. The data from the form were then entered into a computer database. Clinical information for the study was from the database and chart records. Raynaud's phenomenon was defined as sudden reversible white pallor of acral structures, which typically is followed by color changes to purple then to red [6]. The protocol was approved by the Institutional Review Board (IRB). This study meets and is in compliance with all ethical standards in medicine. Informed consent, including the publication of the study, was obtained from all patients according to the Declaration of Helsinki.

\section{Immunoprecipitation}

Autoantibodies in sera from the initial visit of each patient were screened by immunoprecipitation (IP) using ${ }^{35} \mathrm{~S}$-methionine labeled $\mathrm{K} 562$ cell extract. AntiRNAP III were determined using reference sera [4]. Specificity of autoantibodies was determined using previously described reference sera [7].

\section{Immunofluorescent antinuclear antibodies}

Immunofluorescent antinuclear/cytoplasmic antibodies (HEp-2 ANA slides; INOVA Diagnostics, San Diego, CA, USA) were tested using a 1:80-diluted human serum and DyLight 488 donkey IgG $\mathrm{F}\left(\mathrm{ab}^{\prime}\right)_{2}$ anti-human IgG (gamma-chain specific, 1:200 dilution; Jackson ImmunoResearch Laboratories, Inc., West Grove, PA, USA) [8].

\section{ELISA}

Sera were tested for IgG anti-RNAP III antibodies using a commercial ELISA kit (QUANTA Lite ${ }^{\circledR}$ RNA Pol III, INOVA Diagnostics) following the manufacturer's instruction.

\section{Statistical analysis}

Data between groups were compared by the MannWhitney test using Prism 5.0 for Macintosh (GraphPad Software, Inc., San Diego, CA, USA). $P<0.05$ was considered significant.

\section{Results}

Autoantibodies to RNA polymerase I/III were found in 21 patients (1.1\% of 1,966); 18 Caucasians, 2 African Americans, and 1 of mixed ethnic background. Sixteen of 21 cases had a diagnosis of SSc at the initial visit while 5 did not (Table 1). In the Caucasian patients, 14 out of 18 were diagnosed as having SSc at the initial visit. Four patients (cases 1, 3, 4, 5) did not fulfill the SSc criteria at the initial visit when serum anti-RNAP I/ III antibodies were detected. Two African American patients had a diagnosis of SSc at the initial visit. A patient of mixed ethnic background did not meet the American College of Rheumatology (ACR) criteria at the initial visit (case 2). Patients who did not meet SSc criteria at the initial evaluation are summarized below and in Table 1. A brief history of an additional case 6, in which sclerodermatous change was followed by RP two years later, is also described.

Case 1: A fifty-year-old female was hospitalized for shortness of breath and chest pain in March 2000. Numbness in her left second digit also developed and 
Table 1 Five anti-RNAP III positive cases that were not classified as SSc at their initial visit

\begin{tabular}{|c|c|c|c|c|c|c|c|}
\hline & \multicolumn{2}{|c|}{1} & \multicolumn{2}{|c|}{2} & \multirow[t]{2}{*}{3} & \multirow[t]{2}{*}{4} & \multirow[t]{2}{*}{5} \\
\hline & Initial & $f / u$ & Initial & $f / u$ & & & \\
\hline Diagnosis & Vasculitis? & SSC & $\begin{array}{c}\text { Poly } \\
\text {-arthritis }\end{array}$ & SSC & Sine SSc? & ILD & Sjögren's syndrome \\
\hline Race/gender & \multicolumn{2}{|c|}{ WF } & \multicolumn{2}{|c|}{ Mixed F } & WF & WF & WF \\
\hline $\begin{array}{l}\text { Anti-RNAP III } \\
\text { ELISA (u) }\end{array}$ & 16 & NA & 99 & 127 & 8 & $\begin{array}{l}15 \\
\text { to } 47\end{array}$ & 42 \\
\hline Proximal scleroderma & N & $\begin{array}{c}\mathrm{Y} \\
<10 \mathrm{mo}\end{array}$ & N & $\begin{array}{c}Y \\
3 \mathrm{mo}\end{array}$ & N & N & N \\
\hline Sclerodactyly & N & $\begin{array}{c}Y \\
<10 \mathrm{mo}\end{array}$ & N & $\begin{array}{c}Y \\
2 \mathrm{mo}\end{array}$ & N & N & N \\
\hline Pitting scar & N & N & N & N & $\mathrm{N}$ & $\mathrm{N}$ & N \\
\hline ILD & N & N & N & N & N & Y & N \\
\hline Raynaud's phenomenon & N & $?$ & N & $\begin{array}{c}Y \\
6 \mathrm{mo}\end{array}$ & $\begin{array}{c}Y \\
\text { For } 10 y\end{array}$ & N & N \\
\hline Other & & $\begin{array}{c}\text { Renal crisis } \\
10 \mathrm{mo}\end{array}$ & & $\begin{array}{c}\text { Flex.cont } \\
\text { PH }\end{array}$ & ARF (TMA), DCM & & \\
\hline
\end{tabular}

ARF, acute renal failure; DCM, dilated cardiomyopathy; W, white; F, female; Flex cont, flexion contracture; f/u, follow up; ILD, interstitial lung disease; N, no; NA not available; $\mathrm{PH}$, pulmonary hypertension; SSc, systemic sclerosis, scleroderma; TMA, thrombotic microangiopathy; $\mathrm{Y}$, yes

became progressively ischemic and painful, resulting in amputation due to gangrene in April of that year. A hypercoagulable state secondary to malignancy was suspected, but nothing was found. In May 2000, she developed ischemic areas on the tips of the fingers and toes, and was put on anticoagulation therapy. Her ANA (speckled pattern, titer 1:640) and anti-RNAP I/III antibodies were positive but no scleroderma or RP was noted. Prednisolone ( $40 \mathrm{mg} /$ day) was started and her condition was followed at her local clinic. She developed proximal scleroderma and scleroderma renal crisis in March 2001.

Case 2: A 39-year-old female developed polyarthritis involving the metacarpophalangeal joints (MCPs), proximal interphalangeal joints (PIPs), wrists and ankles in August 2004. The initial diagnosis was early synovitis without evidence of systemic rheumatic disease. Rheumatoid factor (RF) and anti-CCP were negative but ANA (speckled pattern, titer 1:160) and anti-RNAP I/III antibodies were positive. She developed sclerodermatous changes in her fingers, forearms and face in October 2004, which rapidly progressed to proximal scleroderma in November. Monthly i.v cyclophosphamide therapy was started, followed by prednisolone ( $50 \mathrm{mg} /$ day) in December. She started having RP in February 2005 and verapamil and bosentan were started. Right heart catheterization in March 2005 suggested mild pulmonary hypertension (PH). Scleroderma progressed to involve the chest, shoulders and abdomen, and flexion contractures of the fingers were noted in March 2005. She was hospitalized in 2008 for anemia caused by gastric antral vascular ectasia (GAVE).

Another very similar case was seen. A 32-year-old Caucasian female was classified as having early synovitis (wrists, MCP, PIP joints) with a positive ANA (speckled and nucleolar pattern). A year later she developed proximal scleroderma and visited the UFCAD. RP developed six months later.

Case 3: A 57-year-old female, who had a 10-year history of RP, visited a clinic for worsening RP in April 2002, when hydroxychroloquine was started. In May 2002 she was hospitalized elsewhere for dyspnea, hypoxia and a nonproductive cough. Pleural effusions and heart failure were found, and her cardiac ejection fraction was $25 \%$. A diagnosis of dilated cardiomyopathy was made. She also developed hypertension and renal dysfunction. Prednisolone $(60 \mathrm{mg} /$ day) and azathioprine were started but the latter was discontinued due to a rash. In July 2002, she developed renal failure and hemodialysis was started. Her kidney biopsy revealed thrombotic microangiopathy. Decreased sensation of her lower left leg was diagnosed as neuropathy. She visited UFCAD in July 2002. No sclerodactyly was noted but one teleangiectasia was found in the digit. ANA (speckled and nucleolar pattern, titer 1:80) and antiRNAP I/III were positive.

Case 4: A 67-year-old female who was followed by a pulmonologist for a three-year history of respiratory symptoms was referred to the UFCAD for a positive ANA (speckled and nucleolar) and ILD. Anti-RNAP I/ III antibodies were positive. She did not have rheumatological symptoms, including arthritis, sclerodermatous changes, or RP at the one-year follow-up.

Case 5: A 68-year-old female was followed for Sjögren's syndrome. She had dry eyes, a dry mouth and a positive salivary gland biopsy but no sclerodermatous skin changes, RP or ILD. She was also positive for ACA by immunofluorescence (Figure 1B panel 5) 


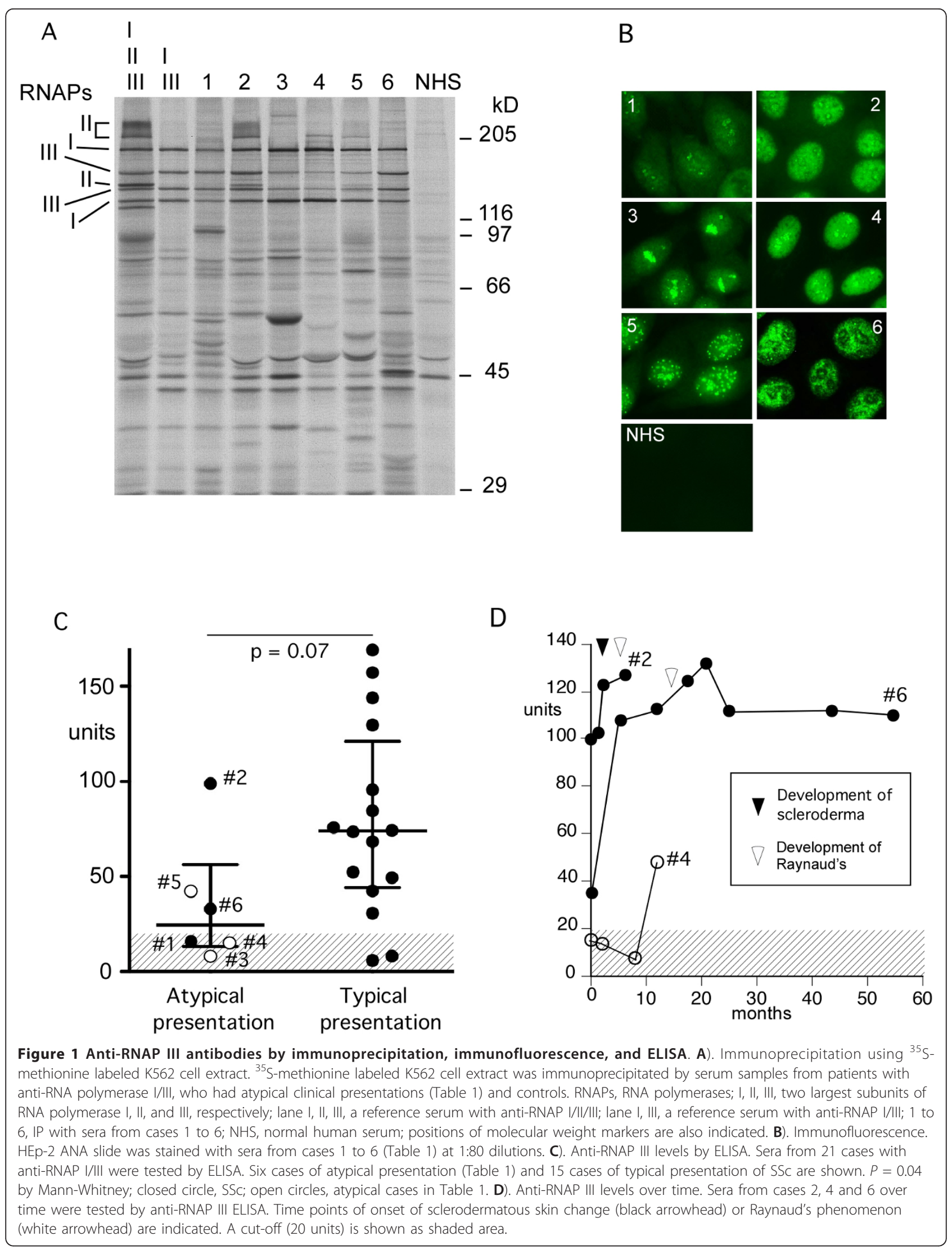


Case 6, (not included in Table 1 since she had a diagnosis of SSc at the initial visit), was a 61-year-old Caucasian female who developed tingling fingertips; carpal tunnel syndrome was suspected. The tingling was followed by swelling and pain in her hands and sclerodermatous skin changes to her forearm. She visited UFCAD and had a diagnosis of diffuse SSc. RP developed two years later.

Two patients (cases 1 and 2), who had anti-RNAP III without SSc but later developed SSc, three patients who did not meet the SSc criteria during observation (cases 3 to 5), and a patient who had SSc without RP and developed RP two years later (case 6), were classified as "atypical presentation" cases. Anti-RNAP III antibodies in these 6 cases were compared with 15 cases of "typical presentation" in which patients had SSc with RP when they visited clinic and serum samples were collected.

Sera from all six cases of "atypical presentation" clearly immunoprecipitated RNAP I and III (Figure 1A). The intensity of RNAP I and III were similar in two patients who developed SSc later (cases 1and 2, lanes 1 and 2 in Figure 1A) and a patient with SSc who developed RP two years after the development of SSc (case 6, lane 6). In contrast, RNAP I was predominant with much weaker RNAP III in three patients, (cases 3 to 5 , lanes 3 to 5 in Figure 1A), who did not have a diagnosis of SSc. RNAP III and II are known to distribute in the nuclei while RNAP I localizes to the nucleoli $[9,10]$. Consistent with their localization patterns, sera from cases 3 to 5 (panels 3 to 5 in Figure 1B) that had predominant RNAP I IP, had dominant nucleolar staining compared with their nuclear staining. Case 5 had ACA in addition (Figure 1B panel 5).

Levels of anti-RNAP III were tested by ELISA comparing cases with atypical presentation vs typical presentation of SSc (Figure 1C). Levels of anti-RNAP III in the former group were lower than those in the latter $(P=$ 0.04 by Mann-Whitney test). Also, anti-RNAP III ELISA negative $(<20$ units) was common in the former ( 3 of 6) vs the latter group ( 1 of $15, P=0.0526$ by the Fisher's exact test). Specifically, 2 of the ELISA negative patients were cases 3 and 4 who had week bands by IP and did not develop scleroderma. Thus, although all cases immunoprecipitated RNAP III, levels of anti-RNAP III by ELISA were lower in cases with atypical clinical presentations compared with those in typical SSc.

Sequential sera from cases 2, 4, and 6 were available for testing by anti-RNAP III ELISA. In case 2, levels of anti-RNAP III were high (99 units) at the initial visit despite complete lack of scleroderma or RP, indicating that anti-RNAP III can be produced prior to clinical manifestations similar to other disease marker autoantibodies. Levels of anti-RNAP III went up when the patient developed sclerodermatous skin changes followed by RP. In case 4, anti-RNAP III became positive while the patient was followed up for ILD, but no clinical changes were observed. In case 6 , the patient had low levels of anti-RNAP III when she visited UFCAD with diffuse SSc but without RP. Her antiRNAP III levels increased from 33 units to 107 units to 112 units prior to the development of RP two years later.

RP is often the first symptom of SSc and may start many years prior to development of SSc [2]. Since cases of anti-RNAP III positive patients who developed sclerodermatous changes prior to RP were noted, the sequence of RP and sclerodermatous changes were reviewed carefully, comparing SSc patients with antiRNAP III vs other specificities in the UFCAD cohort (Table 2 cases 3 to 5 are not included). Almost all of the SSc patients had RP during the course of the disease regardless of the autoantibody specificity. However, only 3 of 17 anti-RNAP III positive patients did not have RP by the time of initial visit $(P=0.07$ vs topo I group, $P=$ 0.01 vs all others combined). When the medical history was carefully reviewed, sclerodermatous changes appeared prior to RP in 31\% (5 of 16) of anti-RNAP III patients while this occurred only in one anti-topo I positive patient in other groups (RNAP III vs topo I, $P=$ 0.03 ; RNAP III vs ACA, $P=0.04$; RNAP III vs others, $P$ $=0.002)$. The development of RP and sclerodactyly were separated by more than one year only in $25 \%$ of antiRNAP III patients vs 50 to $58 \%$ of individuals with other specificities ( $P=0.08$ vs all others). The time between RP and the development of scleroderma was shorter in anti-RNAP III vs ACA group or all others combined ( $P=0.03$ by Mann-Whitney).

\section{Discussion}

Anti-RNAP III antibodies are considered highly specific for the diagnosis of SSc. Among five patients who did not meet the SSc criteria at the initial visit, two developed SSc during follow-up; however, three did not (Table 1). Case 3 had RP and an episode consistent with scleroderma renal crisis and Ccase 4 had ILD. Although their SSc-like features involving internal organs are limited compared with reported cases $[11,12]$, it seems reasonable to suspect that they had a pathogenetic condition similar to systemic sclerosis sine scleroderma, in particular with detection of antiRNAP III. Similar cases of scleroderma renal crisis with minimal or no features of SSc, some with antiRNAP III antibodies, have been reported $[13,14]$. Although we should not classify all ANA-positive acute renal failure or ILD as systemic sclerosis sine scleroderma, identifying SSc-specific autoantibodies may prove useful in understanding the pathogenetic mechanism and selecting treatment options. 
Table 2 Raynaud's phenomenon and autoantibodies in scleroderma patients

\begin{tabular}{lccccc}
\hline & $\begin{array}{c}\text { RNAP III } \\
(\boldsymbol{n}=\mathbf{1 8})\end{array}$ & $\begin{array}{c}\text { Topo I } \\
(\boldsymbol{n}=\mathbf{2 4})\end{array}$ & $\begin{array}{c}\text { ACA } \\
(\boldsymbol{n}=\mathbf{1 5})\end{array}$ & $\begin{array}{c}\text { U3RNP } \\
(\boldsymbol{n}=\mathbf{9})\end{array}$ & $\begin{array}{c}\text { Th/To } \\
(\boldsymbol{n}=\mathbf{8})\end{array}$ \\
\hline Prevalence of RP & $94 \%(16 / 17)$ & $96 \%(23 / 24)$ & $100 \%(15 / 15)$ & $100 \%(9 / 9)$ & $87 \%(7 / 8)$ \\
$\begin{array}{l}\text { Absence of RP at first visit } \\
\text { (in RP positive cases) }\end{array}$ & $18 \%(3 / 16)^{1,2}$ & $0 \%(0 / 23)^{2}$ & $0 \%(0 / 15)$ & $0 \%(0 / 9)$ & $0 \%(0 / 7)$ \\
Scleroderma prior to RP & $31 \%(5 / 16)^{3,4,5}$ & $4 \%(1 / 23)^{3}$ & $0 \%(0 / 15)^{4}$ & $0 \%(0 / 9)$ & $0 \%(0 / 8)$ \\
RP to scleroderma > 1 y & $25 \%(4 / 16)^{6}$ & $53 \%(10 / 19)$ & $58 \%(7 / 12)$ & $50 \%(4 / 8)$ & $57 \%(4 / 7)$ \\
RP to scleroderma & $1.5 \pm 5.3^{7,8}$ & $4.1 \pm 8.9$ & $5.7 \pm 5.8^{7}$ & $1.1 \pm 1.7$ & $2.6 \pm 3.4$ \\
(year, mean \pm SD) & $(0.2 \pm 1.2)^{9}$ & & & & \\
\hline
\end{tabular}

RP, Raynaud's phenomenon

${ }^{1}$ RNAP III vs others, $P=0.01 ;{ }^{2}$ RNAP III vs Topo I, $P=0.07 ;{ }^{3}$ RNAP III vs Topo I, $P=0.03 ;{ }^{4}$ RNAP III vs ACA, $P=0.04 ;{ }^{5}$ RNAP III vs others, $P=0.002 ;{ }^{6}$ RNAP III vs others, $P=0.08 ;{ }^{7}$ RNAP III vs ACA, $P=0.03 ;{ }^{8}$ RNAP III vs others, $P=0.03 ;{ }^{9}$ Value after excluding an outlier that has 21 years interval between RP to scleroderma.

${ }^{1-6}$ by Fisher's exact test, ${ }^{7,8}$ by Mann-Whitney

Detection of anti-RNAP III by ELISA in patients with diagnosis other than SSc was occasionally reported, but most of them were interpreted as false positives based on negative results by IP $[15,16]$. The presence of antiRNAP III confirmed by IP in non-SSc patients, as shown in the present study, was rarely reported $[4,15]$. Several studies compared ELISA and IP to estimate specificity and sensitivity of ELISA. IP positive ELISA negative (false negative) is reported in 4 to $9 \%$ [15-17], while IP negative ELISA positive (false positive) is 12 to $15 \%$ $[15,16]$. Although confirmation of IP negative appears to be incomplete, other study suggests that false positive by ELISA is as low as $~ 2 \%$ [17]. False positives appear to be more common among weakly positive samples or in non-SSc patients $[15,16]$. Our data showed that $19 \%$ (4 of 21, 1 later became positive) of IP positives were ELISA negative (Figure 1). Anti-RNAP III ELISA has been shown to have a good sensitivity and specificity [15-17] and has made the testing for this common SSc antibody widely available. Thus, it significantly helps clinical practice since IP is not available to most clinicians. Nevertheless, it should be kept in mind that there are false positives and false negatives in ELISA. In particular, cautious interpretation will be necessary for weakly positive samples or positives among non-SSc patients. IP should remain the gold standard for antiRNAP III antibody testing.

It is of interest that all three cases of non-SSc antiRNAP III positive patients had predominant RNAP I reactivity with weak RNAP III reactivity and had a strong nucleolar staining that is not always seen in antiRNAP I/III positive SSc patients $[17,18]$. In addition, anti-RNAP III levels increased prior to development of scleroderma or RP in cases 2 and 6, suggesting a correlation between levels of anti-RNAP III and disease activity. One study suggested a link between increasing levels of anti-RNAP III after the initial visit and increasing total skin score and onset of renal crisis over time [15].
Another study reported an association of anti-RNAP III levels and skin score and a negative correlation with a pulmonary function test [19]. These are consistent with the present cases; however, the course of anti-RNAP III during the onset of sclerodermatous skin changes or RP has not been reported previously.

A unique feature observed in anti-RNAP III positive SSc patients was the late development of RP, which has also been suggested [2], but details were not reported. Even if RP appears prior to scleroderma, the interval is often within a year, consistent with the previously reported rapidly progressive nature of SSc in anti-RNAP III positive patients $[2,20]$. The development of RP after the initial visit was observed in only three cases with anti-RNAP III but did not appear in other groups at all, and 31\% (5 of 16) in the antiRNAP III group had scleroderma prior to RP. In contrast, RP preceded scleroderma in most cases of SSc [2]. Sclerodermatous skin without RP is considered characteristic of malignancy-associated pseudoscleroderma [21-23]. However, anti-RNAP III positive SSc should be considered in the differential diagnosis of scleroderma without RP, since $31 \%$ of our anti-RNAP III positive SSc developed sclerodermatous changes prior to RP.

\section{Conclusions}

In summary, anti-RNAP III is highly specific for SSc and related conditions even in an unselected population from a rheumatology clinic. In cases with atypical SSc, dominance of anti-RNAP I and strong nucleolar staining may be seen. The unusual presentation of the occurrence of scleroderma without RP appears to be characteristic of anti-RNAP III positive SSc. In some cases, internal organ involvement, such as renal or lung disease, may precede skin manifestation of SSc, and detection of anti-RNAP III provides useful diagnostic and prognostic information. 


\section{Abbreviations}

ACA: anticentromere antibodies; ACR: American College of Rheumatology; ANA: antinuclear antibodies; GAVE: gastric antral vascular ectasia; ILD: interstitial lung disease; IP: immunoprecipitation; IRB: Institutional Review Board; MCPs: metacarpophalangeal joints; PIPs: proximal interphalangeal joints; PH: pulmonary hypertension; PM/DM: polymyositis/dermatomyositis; RNAP: ribonucleic acid polymerase; RF: rheumatoid factor; RP: Raynaud's phenomenon; SLE: systemic lupus erythematosus; SS: Sjögren's syndrome; SSc: systemic sclerosis: scleroderma; Topo I: topoisomerase I; UFCAD: University of Florida Center for Autoimmune Diseases

\section{Acknowledgements}

We would like to thank Marlene Sarmiento, Annie Chan, and the UF GCRC staff and Matthew Paulus for assistance with clinical data collection. This study was supported by NIH grant R01-AR40391 and M01R00082 from the U.S. Public Health Service and by generous gifts from Lupus Link, Inc. (Daytona Beach, FL, USA) and Mr. Lewis M. Schott to the University of Florida Center for Autoimmune Disease. Publication of this article was funded in part by the University of Florida Open-Access Publishing Fund.

\section{Author details}

'Department of Oral Biology, University of Florida, 1395 Center Drive, Gainesville, FL 32610-0424, USA. ${ }^{2}$ Division of Rheumatology and Clinical Immunology, Department of Medicine, University of Florida, 1600 SW Archer Road, Gainesville, FL 32610-0221, USA. ${ }^{3}$ INOVA Diagnostics, Inc., 9900 Old Grove Road, San Diego, CA, 92131-1638, USA. ${ }^{4}$ Department of Pathology, Immunology, and Laboratory Medicine, University of Florida, 1600 SW Archer Road, Gainesville, FL 32610-0221, USA.

\section{Authors' contributions}

MEK, YL, SJR, JYFC, EKLC, RWB, TTW and MS carried out the immunoassays. $A C$ and MS designed the study. MS performed the statistical analysis. MRB, ESS and WHR enrolled patients for the study and maintained the database AC, MS and EKLC drafted the manuscript. All authors read and approved the final manuscript.

\section{Competing interests}

RWB and TTW are employees of INOVA Diagnostics. All other authors have no competing interests.

Received: 3 March 2011 Revised: 24 May 2011 Accepted: 22 July 2011 Published: 22 July 2011

\section{References}

1. Satoh M, Vazquez-Del Mercado M, Chan EK: Clinical interpretation of antinuclear antibody tests in systemic rheumatic diseases. Mod Rheumatol 2009, 19:219-228.

2. Steen VD: The many faces of scleroderma. Rheum Dis Clin North Am 2008, $34: 1-15$

3. Arbuckle MR, McClain MT, Rubertone MV, Scofield RH, Dennis GJ, James JA, Harley JB: Development of autoantibodies before the clinical onset of systemic lupus erythematosus. N Engl J Med 2003, 349:1526-1533.

4. Satoh M, Ajmani AK, Ogasawara T, Langdon JJ, Hirakata M, Wang J, Reeves WH: Autoantibodies to RNA polymerase II are common in systemic lupus erythematosus and overlap syndrome. Specific recognition of the phosphorylated (IIO) form by a subset of human sera. J Clin Invest 1994, 94:1981-1989.

5. Steen VD: Autoantibodies in systemic sclerosis. Semin Arthritis Rheum 2005, 35:35-42.

6. Suzuki HI, Miyazono K: Emerging complexity of microRNA generation cascades. J Biochem 2011, 149:15-25.

7. Reeves $\mathrm{WH}$, Narain S, Satoh M: Autoantibodies in systemic lupus erythematosus. In Arthritis and Allied Conditions. A Textbook of Rheumatology.. 15 edition. Edited by: Koopman WJ, Moreland LW. Philadelphia, PA: Lippincott Williams and Wilkins; 2005:1497-1521.

8. Yamasaki Y, Narain S, Hernandez L, Barker T, Ikeda K, Segal MS, Richards HB, Chan EK, Reeves WH, Satoh M: Autoantibodies against the replication protein A complex in systemic lupus erythematosus and other autoimmune diseases. Arthritis Res Ther 2006, 8.R111-120.

9. Hirakata M, Okano Y, Pati U, Suwa A, Medsger TAJ, Hardin JA, Craft J: Identification of autoantibodies to RNA polymerase II. Occurrence in systemic sclerosis and association with autoantibodies to RNA polymerases I and III. J Clin Invest 1993, 91:2665-2772.

10. Jones $E$, Kimura H, Vigneron M, Wang Z, Roeder RG, Cook PR: Isolation and characterization of monoclonal antibodies directed against subunits of human RNA polymerases I, II, and III. Exp Cell Res 2000, 254:163-172.

11. Poormoghim H, Lucas M, Fertig N, Medsger TA Jr: Systemic sclerosis sine scleroderma: demographic, clinical, and serologic features and survival in forty-eight patients. Arthritis Rheum 2000, 43:444-451.

12. Fischer A, Meehan RT, Feghali-Bostwick CA, West SG, Brown KK: Unique characteristics of systemic sclerosis sine scleroderma-associated interstitial lung disease. Chest 2006, 130:976-981.

13. Phan TG, Cass A, Gillin A, Trew P, Fertig N, Sturgess A: Anti-RNA polymerase III antibodies in the diagnosis of scleroderma renal crisis sine scleroderma. J Rheumatol 1999, 26:2489-2492.

14. Canet JJ, Castane J, Alvarez M, Nava JM, Llibre J: Scleroderma renal crisis sine scleroderma. Nephron 2002, 90:119-120.

15. Kuwana M, Okano Y, Pandey JP, Silver RM, Fertig N, Medsger TA Jr: Enzyme-linked immunosorbent assay for detection of anti-RNA polymerase III antibody: analytical accuracy and clinical associations in systemic sclerosis. Arthritis Rheum 2005, 52:2425-2432.

16. Satoh $T$, Ishikawa $O$, Ihn H, Endo H, Kawaguchi $Y$, Sasaki T, Goto D, Takahashi K, Takahashi H, Misaki Y, Mimori T, Muro Y, Yazawa N, Sato S, Takehara K, Kuwana M: Clinical usefulness of anti-RNA polymerase III antibody measurement by enzyme-linked immunosorbent assay. Rheumatology (Oxford) 2009, 48:1570-1574.

17. Parker JC, Burlingame RW, Webb TT, Bunn CC: Anti-RNA polymerase III antibodies in patients with systemic sclerosis detected by indirect immunofluorescence and ELISA. Rheumatology (Oxford) 2008, 47:976-979.

18. Yamasaki Y, Honkanen-Scott M, Hernandez L, Ikeda K, Barker T, Bubb MR, Narain S, Richards HB, Chan EK, Reeves WH, Satoh M: Nucleolar staining cannot be used as a screening test for the scleroderma marker anti-RNA polymerase I/III antibodies. Arthritis Rheum 2006, 54:3051-3056.

19. Nihtyanova SI, Parker JC, Black CM, Bunn CC, Denton CP: A longitudinal study of anti-RNA polymerase III antibody levels in systemic sclerosis. Rheumatology (Oxford) 2009, 48:1218-1221

20. Cavazzana I, Angela C, Paolo A, Stefania Z, Angela T, Franco F: Anti-RNA polymerase III antibodies: a marker of systemic sclerosis with rapid onset and skin thickening progression. Autoimmun Rev 2009, 8:580-584.

21. Querfeld C, Sollberg S, Huerkamp C, Eckes B, Krieg T: Pseudoscleroderma associated with lung cancer: correlation of collagen type I and connective tissue growth factor gene expression. Br J Dermatol 2000, 142:1228-1233.

22. Fujii T, Mimori T, Kimura N, Satoh S, Hirakata M: Pseudoscleroderma associated with transforming growth factor beta1-producing advanced gastric carcinoma: comment on the article by Varga. Arthritis Rheum 2003, 48:1766-1767; author repy 1767-1768.

23. Kikuchi K, Hoashi T, Yazawa N, Tamaki K: Pseudoscleroderma associated with cancer. Clin Exp Dermatol 2006, 31:381-383.

\section{doi:10.1186/ar3422}

Cite this article as: Ceribelli et al: Atypical clinical presentation of a subset of patients with anti-RNA polymerase III - non-scleroderma cases associated with dominant RNA polymerase I reactivity and nucleolar staining. Arthritis Research \& Therapy 2011 13:R119.

\section{Submit your next manuscript to BioMed Central and take full advantage of:}

- Convenient online submission

- Thorough peer review

- No space constraints or color figure charges

- Immediate publication on acceptance

- Inclusion in PubMed, CAS, Scopus and Google Scholar

- Research which is freely available for redistribution 